\title{
Lung MRI in Children: The Road Less Travelled
}

\author{
Kushaljit Singh Sodhi ${ }^{1}$ \\ ${ }^{1}$ Department of Radio-diagnosis, Postgraduate Institute of Medical \\ Education and Research (PGIMER), Chandigarh, India
}

\begin{abstract}
Address for correspondence Kushaljit Singh Sodhi, MD, PhD, FICR, Department of Radio-diagnosis, Postgraduate Institute of Medical Education and Research (PGIMER), Sector-12, Chandigarh, India (e-mail: sodhiks@gmail.com).
\end{abstract}

Abstract
Keywords
- children
- lung
- magnetic resonance
imaging
- pediatric

\section{Introduction}

Chest radiograph is the most common radiological investigation performed worldwide in children. It is considered appropriate and adequate in most of the clinical conditions involving the respiratory system. However, computed tomography $(\mathrm{CT})$ of the chest is often performed wherever detailed anatomical information is required and in some specific clinical indications. It provides additional information but involves radiation exposure, which can have detrimental effects on children. ${ }^{1}$ Magnetic resonance imaging (MRI) of the lungs has been underutilized in daily clinical radiological practice. Advances in MRI technology in the past decade have changed the way we look at the lungs and offer comparative radiological information as $\mathrm{CT}$ in some specific clinical indications. ${ }^{2-4}$ It has the inherent advantage of being nonionizing and can be repeated in children without any radiation risks. This article aims to provide a short review of indications, protocols, advantages, and limitations of thoracic MRI in children.

\section{Indications}

Thoracic MRI can now be performed in virtually all pathologies where CT is performed. Indications can be broadly divided into lung parenchymal, mediastinal, and chest wall pathologies (-Figs 1-5). Thoracic MRI has been reported to be of high sensitivity and specificity, nearly at par with CT, in mediastinal masses and cysts, mediastinal lymph nodes, pulmonary embolism, pulmonary nodules larger than $4 \mathrm{~mm}$, bronchiectasis, congenital entities, pleural collections, chest wall tumors, and infections..$^{5-9}$ However, as of now, thoracic MRI is considered inferior to CT in imaging evaluation of interstitial lung diseases, air trapping, pulmonary nodules less than 3 to $4 \mathrm{~mm}$, and calcifications. ${ }^{10,11}$ This is largely due to lower signal resolution in the lungs and susceptibility differences at the interface.

Specifically, thoracic MRI has demonstrated great diagnostic utility as a radiation-free imaging modality in children with infections (tubercular/nontubercular/hydatid), more so in the setting of immunocompromised children and cystic
DOI https://doi.org/

$10.1055 / \mathrm{s}-0041-1729126$ ISSN 0971-3026

\footnotetext{
(C) 2021. Indian Radiological Association

This is an open access article published by Thieme under the terms of the Creative Commons Attribution-NonDerivative-NonCommercial-License, permitting copying and reproduction so long as the original work is given appropriate credit. Contents may not be used for commercial purposes, or adapted, remixed, transformed or built upon. (https://creativecommons.org/licenses/by-nc-nd/4.0/).

Thieme Medical and Scientific Publishers Private Ltd. A-12, Second Floor, Sector -2, NOIDA -201301, India
} 

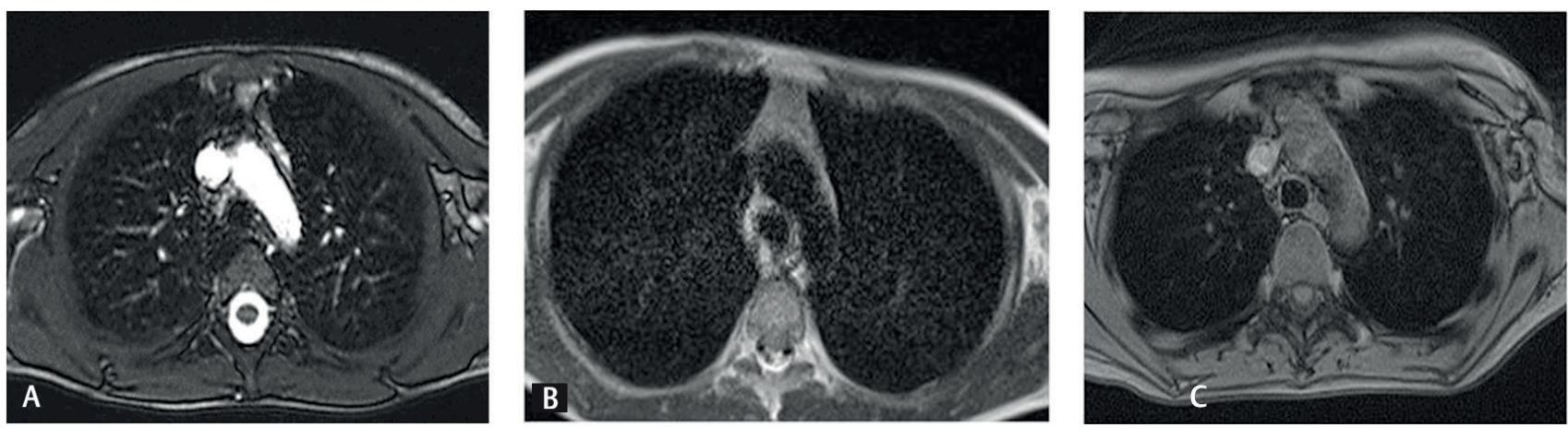

Fig. 1 (a) Normal TRUFI, (b) T2W, and (c) T1 VIBE images at the level of the aortic arch in a 7-year-old boy.
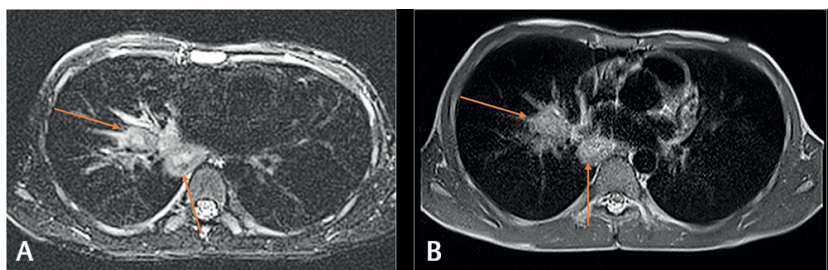

Fig. 2 Necrotic nodes (arrows) on (a) BLADE and (b) T2-weighted images in an 8-year-old boy with febrile neutropenia.

fibrosis. ${ }^{12-16}$ Thoracic MRI is also reported to be useful in the evaluation of large airways in children. ${ }^{17,18}$

- Table 1 provides a complete list of broad categories of indications of thoracic MRI in children.

\section{MRI Protocol}

Typical MR scans in children for lung MRI are performed with either respiratory- or nonrespiratory-gated and nonelectrocardiogram (non-ECG)-gated MRI sequences. Respiratory and ECG gating does offer the advantage of reduced image artifacts and better image quality; however, these gated sequences would entail much higher scan time and this would risk overall compliance of the child for such a study.

In children, time is the most crucial component. MR sequences should be faster and offer great contrast and higher signal-to-noise ratio. Further, to avoid respiratory artifacts, all sequences can be acquired with the breath-holding technique. All children with the help of their parents/guardians should be first taught appropriate
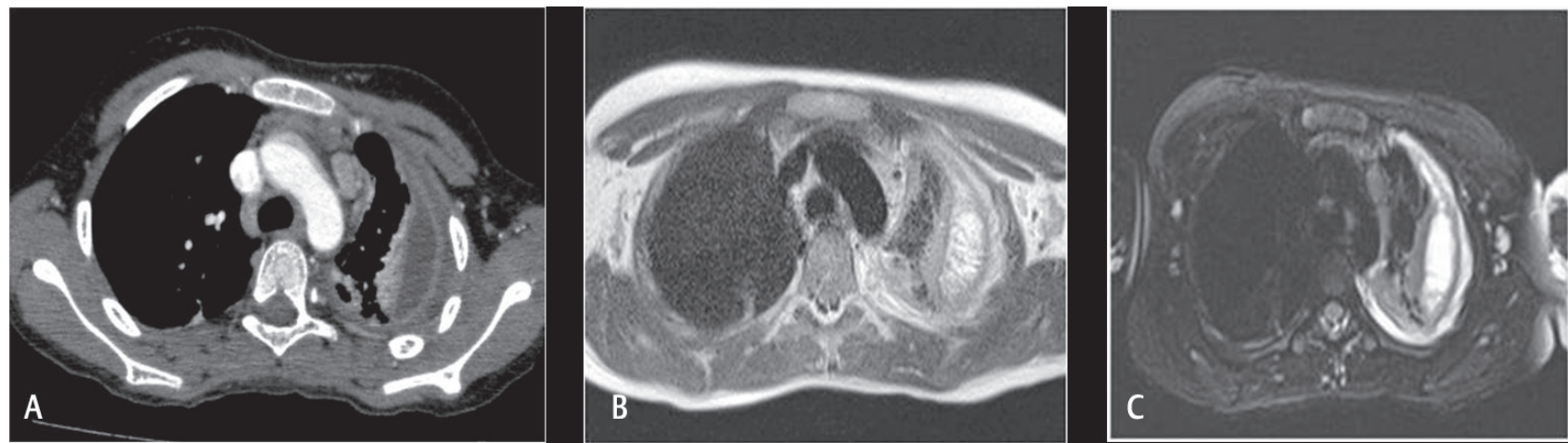

Fig. 3 Left-sided empyema and subsegmental collapse on (a) CT, (b) T2, and (c) BLADE in a 9-year-old girl with fever and chest pain for 4 weeks.
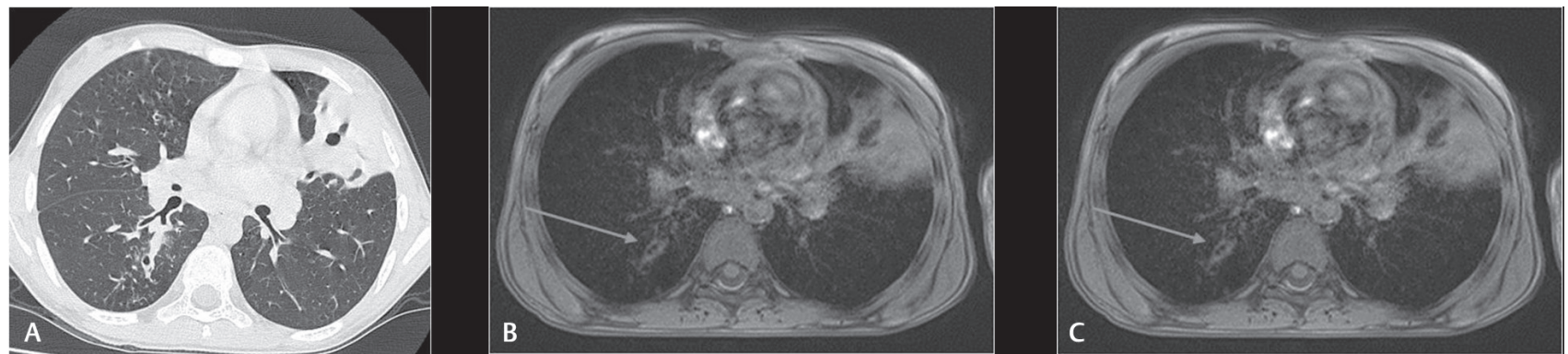

Fig. 4 Consolidation in lingular segments with breakdown on (a) CT, (b) T2, and (c) T1 VIBE in a 12-year-old boy with acute myelogenous leukemia. Mild bronchial wall thickening (arrows) is also noted in the right lower lobe. 


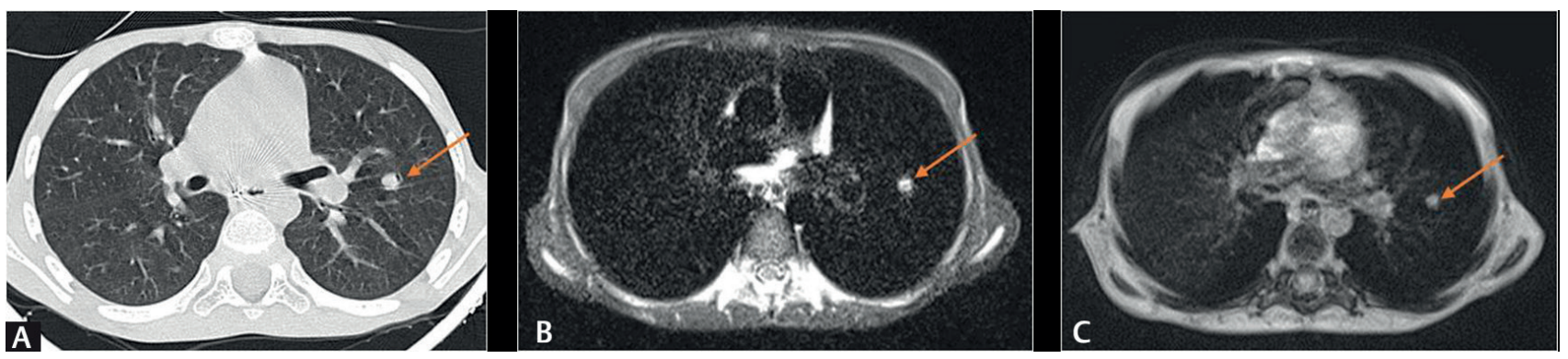

Fig. 5 Small pulmonary nodule in the superior lingula on (a) CT, (b) T2, and (c) T1 VIBE in a 12-year-old boy with acute lymphoblastic leukemia and febrile neutropenia.

minimum breath-holding (10-15 seconds). Children who are sick or cannot hold the breath for short intervals cannot undergo breath-hold MRI sequences, and this is one of the limitations of MRI. However, in children, who cannot hold breath, respiratory triggered free-breathing, T2-weighted sequences can then be utilized. It has to be deployed differently in each child and one size cannot fit all.

Table 1 Typical indications of thoracic MRI in children

\begin{tabular}{|l|l|l|}
\hline $\begin{array}{l}\text { Lung } \\
\text { parenchymal }\end{array}$ & Mediastinal & Chest wall \\
\hline Congenital causes & Lymph nodes & Tumors \\
\hline Infective causes & Masses & Abscesses/infections \\
\hline Interstitial diseases & $\begin{array}{l}\text { Pulmonary } \\
\text { embolism }\end{array}$ & \\
\hline Airway diseases & & \\
\hline Masses/metastasis & & \\
\hline $\begin{array}{l}\text { Vascular } \\
\text { abnormalities }\end{array}$ & & \\
\hline $\begin{array}{l}\text { Pleural diseases } \\
\text { (associated with } \\
\text { parenchymal } \\
\text { disease or only } \\
\text { pleural) }\end{array}$ & & \\
\hline
\end{tabular}

Sedation for smaller children who cannot hold breath or cannot lie on their own for an MRI study would be required. This can be one of the limiting factors for MRI of the lungs in smaller children. Hence, its utility is best advocated for children older than 5 years who can be taught breath-holding and who do not require sedation.

Typical MR sequences include ( - Fig. 1):

- HASTE T2: Half-Fourier-acquisition single-shot turbo spin-echo.

- BLADE T2: Proprietary name for periodically rotated overlapping parallel lines with enhanced reconstruction (PROPELLER) in MR systems.

- TRUFI T2: True fast imaging with steady-state free precession sequence.

- VIBE T1: Volumetric interpolated breath-hold examination.

- Table 2 summarizes the various intrinsic parameters of the sequences used.

These sequences provide most of the required pathological and anatomical information. ${ }^{2-4}$ Additional sequences, such as STIR (short tau inversion recovery) sequence, diffusion-weighted imaging (DWI), and postcontrast imaging, can be added to a given study, on an individual case basis.

Table 2 Intrinsic parameters of our typical MRI sequences

\begin{tabular}{|l|l|l|l|}
\hline Parameters & T2 HASTE & T1 VIBE & TRUFI/SSFP \\
\hline FoV (mm) & 275 & 400 & 300 \\
\hline FoV phase\% & 78.1 & 75 & 85.9 \\
\hline Base resolution & 320 & 384 & 256 \\
\hline Phase resolution (\%) & 80 & 75 & 100 \\
\hline Slice thickness (mm) & 4 & 4 & 4 \\
\hline Phase partial Fourier & $4 / 8$ & $7 / 8$ & Off \\
\hline Pixel size (mm) & $0.7 \times 0.7 \mathrm{~mm}$ & $0.7 \times 0.7 \mathrm{~mm}$ & $0.6 \times 0.6 \mathrm{~mm}$ \\
\hline Distance factor & 20 & 20 & 60 \\
\hline TR (ms) & 500 & 4.06 & 580.23 \\
\hline TE (ms) & 36 & 2.07 & 1.48 \\
\hline Flip-angle (degree) & 146 & 5 & 40 \\
\hline Bandwidth (Hz/pixel) & 781 & 540 & 1,028 \\
\hline iPAT (no. of ref. lines) & $2(1)$ & $2(24)$ & $2(26)$ \\
\hline
\end{tabular}

Abbreviations: FoV, field of view; HASTE, half-fourier-acquisition single-shot turbo spin-echo; iPAT, integrated parallel acquisition techniques; SSFP, steady-state free precession sequence; TR, repetition time; TE, echo time; TRUFI, true fast imaging with steady-state free precession sequence; VIBE, volumetric interpolated breath-hold examination. 


\section{Contrast Administration in Lung MRI}

Contrast is not required to be administered in all routine thoracic MRI cases. However, as per indication, and on a case basis, contrast can be given, for example, in mediastinal masses or suspected abscess formation. Few MR sequences (steady-state free precession [SSFP]/TRUFI) depict inherent vascular contrast in mediastinal vessels without the need for giving any contrast. Anatomy of mediastinal vessels and even pulmonary artery embolism can be detected even without contrast administration.

Lungs are scanned in the axial plane in the craniocaudal direction in all children. The total MRI scanning time can vary from 2 to 14 minutes for all sequences combined. Few authors have reported faster ( 2 minutes) but limited protocol for use in lung MRI in children.,7

\section{Advances in MRI}

Advances in MR technology including newer MRI lung sequences, e.g., ultrashort TE sequence and zero echo time, offer great potential and show promise of yielding very high resolution images at par with high-resolution CT. ${ }^{19-22}$ Both these newer sequences deploy very short TEs to capture magnetic resonance signals from very short intrinsic T2/T2* tissues. Thus, these provide much superior lung parenchymal images.

Diffusion imaging of the lungs is another promising tool, which has as yet not been explored in the pediatric population. DWI with functional MRI has been reported in adults to provide structural as well as functional information in patients with primary antibody deficiencies. ${ }^{23}$ Diffusion imaging of lungs has also been found useful for the detection of inflammatory changes of lung during respiratory tract exacerbations in cystic fibrosis patients. ${ }^{24}$

Functional assessment of the lung parenchyma is another exciting potential application of lung MRI. Functional MRI studies have demonstrated expiratory-inspiratory MR signal-intensity differences, which can differentiate ventilation defects of different types in cystic fibrosis lung disease, and this kind of functional impairment is reported to be directly related to the diseased structural abnormalities. ${ }^{25}$ Dynamic ventilation and perfusion studies are performed and perfusion maps are generated, deploying postprocessing software and algorithms, which can guide patient monitoring in these patients. ${ }^{26}$

\section{Advantages and Limitations of MRI}

Thoracic MRI has its inherent set of advantages and limitations, which have been summarized in tabulated form in - Table 3. It offers a radiation-free alternative to CT scan in most of the common pathologies and provides both anatomic and functional information in one setting. ${ }^{15,26,27}$ It is a particularly more attractive option in children in whom repeated follow-up imaging (e.g., cystic fibrosis) is required as a part of the management protocol. In smaller children, where it
Table 3 Advantages and limitations of lung MRI

\begin{tabular}{|c|c|}
\hline Advantages & Limitations \\
\hline Lack of ionizing radiations & Higher cost factor \\
\hline \multirow{2}{*}{$\begin{array}{l}\text { Can be repeated: no safety } \\
\text { issues of radiations for fol- } \\
\text { low-up in younger children }\end{array}$} & Not widely available \\
\hline & \multirow{3}{*}{$\begin{array}{l}\text { Smaller children would } \\
\text { require sedation }\end{array}$} \\
\hline Higher soft-tissue contrast & \\
\hline \multirow{2}{*}{$\begin{array}{l}\text { Vascular information can be } \\
\text { obtained without administra- } \\
\text { tion of contrast }\end{array}$} & \\
\hline & \multirow{2}{*}{$\begin{array}{l}\text { More image artifacts and } \\
\text { poor image quality if child } \\
\text { not compliant }\end{array}$} \\
\hline \multirow{4}{*}{$\begin{array}{l}\text { Provides both anatomical and } \\
\text { functional information in one } \\
\text { setting }\end{array}$} & \\
\hline & Less spatial resolution overall \\
\hline & $\begin{array}{l}\text { Lower sensitivity in smaller } \\
\text { nodules }(<4 \mathrm{~mm}) \text {, cysts, } \\
\text { air trapping, peripheral } \\
\text { bronchiectasis }\end{array}$ \\
\hline & $\begin{array}{l}\text { Lower sensitivity in small } \\
\text { calcifications }\end{array}$ \\
\hline
\end{tabular}

is not considered safe to administer MR contrast, MRI provides vascular information even without administration of gadolinium-based contrast and specific MR sequences (SSFP) can be performed in these clinical settings.

\section{Conclusion}

Lung MRI in children is difficult and challenging due to low signal generated from lungs, relatively longer imaging times, motion artifacts, and nonuniform cooperation by children. However, it offers a radiation-free alternative to CT, and can provide both anatomical and functional assessment of chest in the same sitting. Lung MRI can be utilized in many specific clinical indications, e.g., pulmonary infections in immunocompromised children, lymph node evaluation, cystic fibrosis, and follow-up imaging in children. CT, however, still remains the investigation of choice in evaluation of interstitial lung disease, smaller airways, and pulmonary micronodules. It is important to optimize all cases of lung MRI in children to reduce MRI scan time with judicious planning and adapting MR protocols and get MR images of high quality.

\section{Financial Support and Sponsorship \\ Nil.}

\section{Conflict of Interest}

The authors declare no conflict of interest.

\section{References}

1 Sodhi KS, Lee EY. What all physicians should know about the potential radiation risk that computed tomography poses for paediatric patients. Acta Paediatr 2014;103(8):807-811

2 Kapur S, Bhalla AS, Jana M. Pediatric chest MRI: a review. Indian J Pediatr 2019;86(9):842-853

3 Sodhi KS, Khandelwal N, Saxena AK, et al. Rapid lung MRI in children with pulmonary infections: time to change our diagnostic algorithms. J Magn Reson Imaging 2016;43(5): 1196-1206 
4 Biederer J, Mirsadraee S, Beer M, et al. MRI of the lung (3/3)-current applications and future perspectives. Insights Imaging 2012;3(4):373-386

5 Zirpoli S, Munari AM, Primolevo A, et al. Agreement between magnetic resonance imaging and computed tomography in the postnatal evaluation of congenital lung malformations: a pilot study. Eur Radiol 2019;29(9):4544-4554

6 Liszewski MC, Görkem S, Sodhi KS, Lee EY. Lung magnetic resonance imaging for pneumonia in children. Pediatr Radiol 2017;47(11):1420-1430

7 Sodhi KS, Khandelwal N, Saxena AK, et al. Rapid lung MRI - paradigm shift in evaluation of febrile neutropenia in children with leukemia: a pilot study. Leuk Lymphoma 2016;57(1):70-75

8 Rana P, Sodhi KS, Bhatia A, Saxena AK, Suri D, Singh S. Diagnostic accuracy of 3-T lung magnetic resonance imaging in human immunodeficiency virus-positive children. Pediatr Radiol 2020;50(1):38-45

9 Yikilmaz A, Koc A, Coskun A, Ozturk MK, Mulkern RV, Lee EY. Evaluation of pneumonia in children: comparison of MRI with fast imaging sequences at $1.5 \mathrm{~T}$ with chest radiographs. Acta Radiol 2011;52(8):914-919

10 Sodhi KS, Sharma M, Lee EY, et al. Diagnostic utility of 3T lung MRI in children with interstitial lung disease: a prospective pilot study. Acad Radiol 2018;25(3):380-386

11 Sodhi KS, Gupta P, Shrivastav A, et al. Evaluation of $3 \mathrm{~T}$ lung magnetic resonance imaging in children with allergic bronchopulmonary aspergillosis: pilot study. Eur J Radiol 2019;111:88-92

12 Sodhi KS, Bhalla AS, Mahomed N, Laya BF. Imaging of thoracic tuberculosis in children: current and future directions. Pediatr Radiol 2017;47(10):1260-1268

13 Ozcan HN, Gormez A, Ozsurekci Y, et al. Magnetic resonance imaging of pulmonary infection in immunocompromised children: comparison with multidetector computed tomography. Pediatr Radiol 2017;47(2):146-153

14 Sodhi KS, Sharma M, Saxena AK, Mathew JL, Singh M, Khandelwal N. MRI in thoracic tuberculosis of children. Indian J Pediatr 2017;84(9):670-676

15 Wielpütz MO, von Stackelberg O, Stahl M, et al. Multicentre standardisation of chest MRI as radiation-free outcome measure of lung disease in young children with cystic fibrosis. J Cyst Fibros 2018;17(4):518-527

16 Singh R, Garg M, Sodhi KS, et al. Diagnostic accuracy of magnetic resonance imaging in the evaluation of pulmonary infections in immunocompromised patients. Pol J Radiol 2020; 85:e53-e61

17 Liszewski MC, Ciet P, Lee EY. MR imaging of lungs and airways in children: past and present. Magn Reson Imaging Clin N Am 2019;27(2):201-225

18 Liszewski MC, Ciet P, Sodhi KS, Lee EY. Updates on MRI evaluation of pediatric large airways. AJR Am J Roentgenol 2017;208(5):971-981

19 Willmering MM, Robison RK, Wang H, Pipe JG, Woods JC. Implementation of the FLORET UTE sequence for lung imaging. Magn Reson Med 2019;82(3):1091-1100

20 Chassagnon G, Martin C, Ben Hassen W, et al. High-resolution lung MRI with ultrashort-TE: 1.5 or 3 Tesla? Magn Reson Imaging 2019;61:97-103

21 Bae K, Jeon KN, Hwang MJ, et al. Comparison of lung imaging using three-dimensional ultrashort echo time and zero echo time sequences: preliminary study. Eur Radiol 2019;29(5):2253-2262

22 Torres L, Kammerman J, Hahn AD, et al. Structure-function imaging of lung disease using ultrashort echotime MRI. Acad Radiol 2019;26(3):431-441

23 Milito C, Pulvirenti F, Serra G, et al. Lung magnetic resonance imaging with diffusion weighted imaging provides regional structural as well as functional information without radiation exposure in primary antibody deficiencies. J Clin Immunol 2015;35(5):491-500

24 Ciet P, Bertolo S, Ros M, et al. Detection and monitoring of lung inflammation in cystic fibrosis during respiratory tract exacerbation using diffusion-weighted magnetic resonance imaging. Eur Respir J 2017;50(1):1601437

25 Pennati, F, Roach, DJ, Clancy, JP, et al. Assessment of pulmonary structure- function relationships in young children and adolescents with cystic fibrosis by multi volume proton-MRI and CT. J Magn Reson Imaging 2018;48(2):531-542

26 Kaireit TF, Voskrebenzev A, Gutberlet M, et al. Comparison of quantitative regional perfusion-weighted phase resolved functional lung (PREFUL) MRI with dynamic gadolinium-enhanced regional pulmonary perfusion MRI in COPD patients. J Magn Reson Imaging 2019;49(4):1122-1132

27 Sodhi KS, Bhatia A, Samujh R, Mathew JL, Lee EY. Prospective comparison of MRI and contrast-enhanced MDCT for evaluation of pediatric pulmonary hydatid disease: added diagnostic value of MRI. AJR Am J Roentgenol 2019;212(5):982-987 